Urol Nephrol

July 2018 Vol.:5, Issue:2

(C) All rights are reserved by Yddoussalah, et al.

\section{Testicular Epidermoid Cyst: Case Report and Literature Review}

\begin{abstract}
Keywords: Case report; Testicular tumor; Benign tumor; Epidermoid cyst; Conservative treatment

Abstract

Introduction: Testicular epidermoid cysts are extremely uncommon. They are considered as benign tumors. Although an inguinal orchiectomy is the standard procedure for testicular tumors, if the presence of an epidermoid cyst is suspected, a conservative treatment may be attempted.

Case Presentation: We report here the case of a testicular epidermoid cyst in a 28-year-old man, who consulted for infertility. Physical examination shows the presence of a hard swelling on the left testicle surface, suggesting the presence of a mass. Enucleation was performed and no tumor recurrence was observed during the 6 months of follow-up.

Conclusion: Thus, we will expose, through this observation and the literature, the clinicopathological characteristics of this rare testicular tumor and the different therapeutic aspects. This work has been reported in line with the SCARE criteria [1].
\end{abstract}

\section{Introduction}

Testicular epidermoid cysts (TEC) are extremely rare and account for less than $1 \%$ of all testicular tumors. Orchiectomy is the standard treatment for clinically suspected malignant testicular tumors. On the other hand, if an intra-testicular benign lesion is suspected, conservative treatment may be offered to the patient. We report a new observation whose interest lies in the benignity of the lesion, but also in its association with sterility of the couple.

\section{Case Presentation}

A 28-year-old male patient, with no medical history, consulted for primary infertility after 3 years of marriage. His physical examination showed a hard swelling on the left testicle surface, suggesting the presence of a mass, without neither pain nor inflammatory signs. The contralateral testicle was normal. The patient does not report the presence of LUTS (lower urinary tract symptoms) or other associated signs, and in particular, tuberculosis signs.

Two spermograms performed one month apart showed oligoasthenoteratospermia (OATS). The measurement of tumor markers was normal.

Ultrasound showed a rounded, intra-parenchymal formation with a heterogeneous and hyperechogenic center, associated with peripheral calcifications (Figure 1). No anomaly was objectified in the contralateral testicle.

Given the ultrasound appearance of the testicular lesion, conservative surgery has been proposed to the patient. Thus, exploration by left inguinal incision was performed with first clamping of the spermatic cord (Figure 2). The testicular mass was palpated and enucleated with macroscopically healthy margins (Figure 3 ). The postoperative course was simple.

Macroscopic anatomic and pathological examination showed,
Journal of

\section{Urology \& Nephrology}

\section{Othmane Yddoussalah ${ }^{1,2 *}$, Mohammed Alae Touzani ${ }^{1,2}$, Tariq Karmouni ${ }^{1,2}$, Khalid El Khader ${ }^{1,2}$ Abdellatif Koutani ${ }^{1,2}$ and Ahmed Ibn Attya Andalouss $i^{1,2}$}

${ }^{I}$ Faculty of Medicine and Pharmacy, Mohammed $V$ University, Rabat, Morocco

${ }_{2}^{2}$ Urology Department, Ibn Sina Teaching Hospital, Rabat, Morocco Address for Correspondence

Othmane Yddoussalah, Résidence Wifaq, Immeuble 2, Appt 7, Hay Riad (Secteur 21)-Rabat, Morocco, Tel: +212 6685178 70; E-mail: yddoussalah@gmail.com

Submission: 18 June, 2018

Accepted: 30 June, 2018

Published: 06 July, 2018

Copyright: $\odot 2018$ Yddoussalah O, et al. This is an open access article distributed under the Creative Commons Attribution License, which permits unrestricted use, distribution, and reproduction in any medium, provided the original work is properly cited.

after opening of the cyst, a whitish, lumpy content arranged in concentric lamellae measuring $15 \mathrm{~mm}$ of long axis (Figure 4). The microscopic study confirmed the benign lesion and made the diagnosis of epidermoid cyst of the left testicle. At 24-month followup, no tumor recurrence, neither on clinical examination nor ultrasound has been found.

\section{Discussion}

Testicular cancer is relatively rare, with only about 5,500 new cases reported per year in the United States [2]

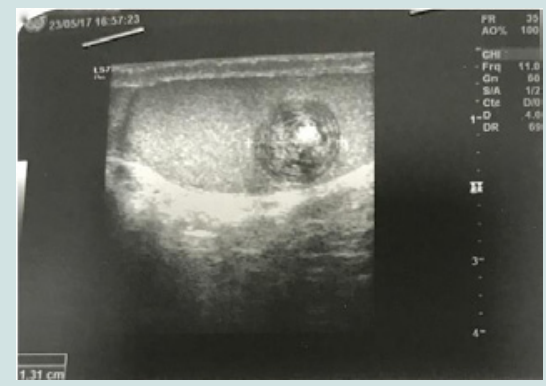

Figure 1: Ultrasound.

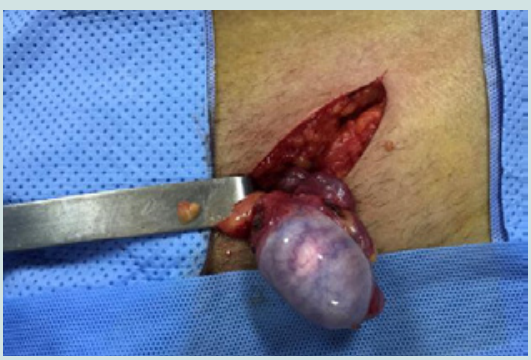

Figure 2: Macroscopical appearance of the testicle. 


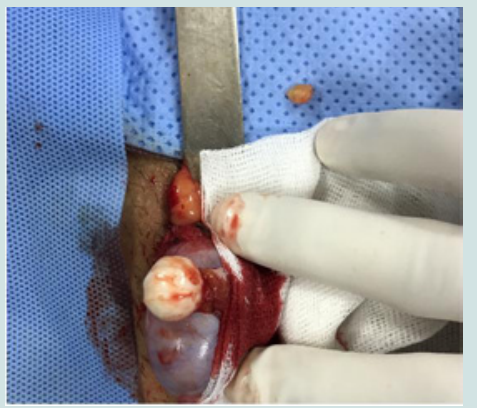

Figure 3: Macroscopical appearance of the tumor.

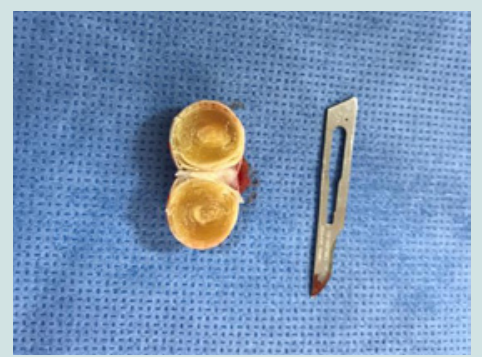

Figure 4: Macroscopical internal appearance of the tumor.

Epidermoid cysts are benign lesions often located in the skin and very rarely intra-testicular. Histologically, they have the same characteristics as cutaneous lesions. Since the first description of Dockerty and Priestley in 1942, about 300 cases of testicular epidermoid cysts have been reported in the literature.

These are generally unique lesions affecting a young population between 20 and 40 years old [3]. Rare cases of bilateral involvement and in children have been reported.

Clinically, the lesions are usually firm, well circumscribed, small, single, painless, and sometimes difficult to distinguish from a malignant lesion. The measurement of testicular tumor markers is always normal [4].

Ultrasound shows a characteristic image of the TEC, a "target" image. The cystic wall can be either hypoechoic or hyperechoic due to focal calcifications. The center of the cyst is heterogeneous with hyper or hypoechoic zones attributable to the density of the keratin lamellae that compose it [5-7]. Since the advent of MRI, the images are more characteristic of the TEC, which makes it possible to differentiate from other solid intratesticular lesions. The typical appearance is the "bull's eye". The wall and the centre of the cyst are in hypo-signal in T1 and T2 weighting. Between these two zones, there is in T1 and T2 weighting a hyper-signal that corresponds to isolated and degenerated epithelial cells (rich in water and fat) [6]. There is no enhancement of the signal after injection of contrast dye because it is an avascular structure.

The histological diagnosis was established by PRICE in 1969, the tumoral mass must necessarily gather five anatomo-pathological criteria so that one can speak of isolated TEC [8]: the TEC must be in the testicular parenchyma, it wall should be constituted by fibrous tissue and contain squamous epithelium, it should contain keratin lamellae or amorphous material, no teratomatous elements or cutaneous appendages should be found in the cyst wall or adjacent to testicular parenchyma, and no hyaline element (remnant of a germ cell tumor) should be found in the adjacent testicular parenchyma.

The recommended treatment is surgical with an inguinal approach. Conservative surgery for TCE has all its place given the psychological and reproductive consequences of total orchiectomy for a benign pathology. Recently, several authors have proposed partial resection or enucleation as the treatment of choice in adults and children $[4,9]$. Heidenreich et al. have, in a review of the literature including 300 cases treated by a conservative approach, objectified no case of local recurrence or distant metastasis [10]. Ross et al. estimate that conservative surgery is more appropriate in the pediatric population because of the higher percentage of benign testicular tumors compared to adults and the risk of contralateral testicular disease (e.g. torsion) is much higher in the child [9]. Some authors report an improvement in long-term fertility even in patients with oligospermia or contralateral disease, after conservative testicular surgery. However, we must keep in mind the risk of infertility induced by testicular surgery secondary to the rupture of the bloodtestis barrier and the immunological consequences demonstrated on the animal model $[11,12]$. However, recently, studies have shown that anti-sperm antibodies, found in sperm after testicular biopsy or surgical enucleation, do not affect reproductive outcomes (fertilization and clinical pregnancy rates) [13].

Postoperative monitoring is not codified in TEC.

\section{Conclusion}

TEC are rare and benign testicular tumors. Currently, the existing clinical examination and paraclinical tools can guide the diagnosis preoperatively and thus attempt a conservative surgery. Performing biopsy of the adjacent testicular parenchyma is essential. Orchiectomy is mandatory if the extemporaneous examination is not formal.

\section{References}

1. Agha RA, Fowler AJ, Saeta A, Barai I, Rajmohan $S$, et al. (2016) The SCARE Statement: consensus-based surgical case report guidelines. Int J Surg 34: 180-186.

2. Moghe PK, Brady AP (1999) Ultrasound of testicular epidermoid cysts. Br J Radiol 72: 942-945

3. Aguilera TC, Lopez RG, Roca EA, Martin GB, Hernandez RR et al. (2005) Testicular epidermoid cyst: uncommon lesion of difficult preoperative diagnosis. Actas Urol Esp 29: 905-908.

4. Walsh C, Rushton HG (2000) Diagnosis and management of teratomas and epidermoid cyst. Urol Clin North Am 27: 509-518.

5. Eisenmenger M, Lang S, Donner G, Kratzik C, Marberger M (1993) Epidermoid cysts of the testis: organ-preserving surgery following diagnosis by ultrasonography. $\mathrm{Br} \mathrm{J}$ Urol 72: 955-957.

6. Fu YT, Wang HH, Yang TH, Chang SY, Ma CP (1996) Epidermoid cysts of the testis: diagnosis by ultrasonography and magnetic resonance imaging resulting in organ-preserving surgery. Br J Urol 78: 116-118.

7. Menasse F, Barriol D, Demoux R, Prost J, Sault MC et al. (2000) Kyste épidermoïde du testicule. Chirurgie conservatrice a propose de 3 cas. Prog Urol 10: 603-606.

8. Price EB Jr (1969) Epidermoid cysts of the testis: a clinical and pathologic analysis of 69 cases from the testicular tumor registry. J Urol 102: 708-713.

9. Pohl HG, Shukla AR, Metcalf PD, Cilento BG, Retik AB, et al. (2004) 
Citation: Yddoussalah O, Touzani MA, Karmouni T, Khader KE, Koutani A, et al. Testicular Epidermoid Cyst: Case Report and Literature Review. J Urol Nephrol. 2018;5(2): 3.

\section{ISSN: 2380-0585}

Prepubertal testis tumors: actual prevalence rate of histological types. J Uro 172: $2370-2372$

10. Heidenreich A, Engelmann UH, Vietsch HV, Derschum W (1995) Organ preserving surgery in testicular epidermoid cyst. J Urol 153: 1147-1150.

11. Koşar A, Kupeli B, Alcigir G, Ataoglu H, Sarica K. et al. (1999) Immunologic aspect of testicular torsion: detection of antisperm antibodies in contralatera testicle. Eur Urol 36: 640-644.
12. Chehval MJ, Martin SA, Alexander NJ, Winkelmann T (1995) The effect of unilateral injury to the vas deferens on the contralateral testis in immature and adult rats. J Urol 153: 1313-1315.

13. Zini A, Lefebvre J, Kornitzer G, Bissonnette F, Kadoch IJ, et al. (2011) Antisperm antibody levels are not related to fertilization or pregnancy rates after IVF or IVF/ICSI. J Reprod Immunol 88: 80-84. 